\title{
Optimizing telemedicine for providing obstetric and reproductive health care services during COVID-19 pandemic
}

\author{
Vijayan Sharmila ${ }^{1 *}$, Thirunavukkarasu Arun Babu' ${ }^{2}$, Padmapriya Balakrishnan ${ }^{3}$
}

\author{
${ }^{1}$ Department of Obstetrics and Gynaecology, All India Institute of Medical Sciences (AIIMS), Mangalagiri, Andhra \\ Pradesh, India \\ ${ }^{2}$ Department of Paediatrics, All India Institute of Medical Sciences (AIIMS), Mangalagiri, Andhra Pradesh, India \\ ${ }^{3}$ Department of Pathology, Andhra Medical College, Andhra Pradesh, India
}

Received: 02 June 2020

Revised: 10 July 2020

Accepted: 14 July 2020

\section{*Correspondence:}

Dr. Vijayan Sharmila,

E-mail: sharu_jipmer@aiimsmangalagiri.edu.in

Copyright: ( ) the author(s), publisher and licensee Medip Academy. This is an open-access article distributed under the terms of the Creative Commons Attribution Non-Commercial License, which permits unrestricted non-commercial use, distribution, and reproduction in any medium, provided the original work is properly cited.

\begin{abstract}
The coronavirus disease (COVID-19) outbreak was first declared in China in December 2019, and WHO declared the outbreak as a pandemic on 11 March 2020. A fast-rising number of confirmed cases has been observed in countries across all continents over the past few months. This pandemic is forcing government of nations to take strict measures such as complete lockdown and curfews to ensure that citizens stay at home, as a measure to contain the spread of infection. Health sector is facing challenges such as high demand for doctors, limited resources of personal protective equipment and challenges in providing in-person consultation due to fear of spread of infection. Telemedicine is making positive contribution to healthcare during this pandemic, as it caters to health care of people in wide geographical area without risk of acquiring COVID-19. Telemedicine is defined as the provision of health care services by health care professionals, using technology to exchange information. A broad range of obstetric and reproductive health services can be offered via telemedicine. Telemedicine can help pregnant women to maintain high quality of care during this pandemic without compromising on the perinatal outcome. It allows for constant interaction between patient and doctor throughout pregnancy, thereby ensuring that the goals of antenatal care are fulfilled, while limiting unnecessary travel and time away from work and family. This article mainly concentrates on the essential obstetric and reproductive health care services that can be provided by telemedicine during COVID-19 pandemic.
\end{abstract}

Keywords: COVID-19, Obstetric care, Reproductive health care, Telemedicine

\section{INTRODUCTION}

The world is currently battling a healthcare emergency in the form of novel Corona virus disease (COVID-19) caused by severe acute respiratory syndrome coronavirus 2 (SARS-CoV-2). COVID- 19 outbreak first occurred in Wuhan, Hublei Province of China. ${ }^{1}$ World Health Organization (WHO), initially declared as a Public Health Emergency of International Concern (PHEIC) on 30 January 2020, and later declared the new coronavirus as a global pandemic on March 11, 2020 and called for government action to halt the spread of the virus. Since then, there is a fast surge in the number of confirmed cases in several countries across all continents. Health sector is facing challenges in providing in-person consultation for patients due to risk of easy spread of COVID-19, high demand of doctors and limited resources of personal protective equipment. Telemedicine is making a very positive contribution to healthcare during the pandemic, as it can provide health care to people in wide 
geographical area with no risk of acquiring COVID-19. Telemedicine essentially includes diagnosis and treatment of patients at a distance by using telecommunication technology. The term "telemedicine" literally means 'healing at a distance', referring to provision of remote virtual healthcare with the healthcare provider and the patient physically in different locations.

World Health Organization (WHO) has defined telemedicine as, "the delivery of healthcare services, where distance is a critical factor, by all healthcare professionals using information and communication technologies, for the exchange of valid information for diagnosis, treatment and prevention of disease and injuries, research and evaluation, and for the continuing education of healthcare providers, all in the interests of advancing the health of individuals and their communities." Information and communication technology is used for taking history, reasonable relevant clinical examination and review of investigations. Telemedicine-enabled devices such as the thermometer, torch, stethoscope, BP apparatus, ECG, glucometer etc., can transmit patient's data from remote location. The provisional diagnosis can be communicated to the patient electronically along with specific advice and medical prescription.

\section{HISTORICAL BACKGROUND}

The earliest published data on telemedicine was the transmission of ECG over telephone lines in the beginning of 20th century. ${ }^{1}$ Inventions like the telegraph and telephone played a vital role in the evolution of the modern telemedicine. ${ }^{2}$ The first known record of realtime video consultation happened in 1959, when interactive telemedicine was used to transmit neurological examinations by the doctors at University of Nebraska. ${ }^{3}$

NASA first used telemedicine services for disaster management during the Mexico City earthquake and the Soviet Armenia earthquake in 1985 and 1988 respectively, when all other modes of communication were disrupted. ${ }^{4}$ Over the past few decades, advances in wireless broadband technology has lead to ubiquitous use of computers, smart phones and internet. ${ }^{5}$ The modern telemedicine makes use of self-owned gadgets such as computing devices, smart phones and cameras of the patients and doctors to gather clinical data, transfer of radiological, lab investigations, patient education videos, and real-time audio and video consultations. ${ }^{2}$

\section{TYPES OF TELEMEDICINE SERVICES}

Technical requirements for telemedicine services include hardware, software, internet connectivity along with acquisition, storage, retrieval, and display facilities in the gadgets used for this purpose.

Telemedicine can facilitate a broad range of interactions, using different modalities and devices. ${ }^{1}$ Interaction between health care provider and patient provides healthcare even to the unreachable population by giving them direct access to medical services. Interaction between two or more health care providers provide easier access to specialty care, referral and consultation services.

Modalities of information transmission include the real time or synchronous telemedicine in which the sender and receiver both are online at the same point of time and 'live' transfer of information occurs and store-andforward or asynchronous telemedicine in which the sender stores the information databases and sends it to the receiver at a convenient point of time, and the receiver can review the data according to his/her convenience.

Remote monitoring type of telemedicine (self-monitoring or self-testing) in which technological devices are used to monitor the health of a patient remotely. Interaction can be via telephonic consultation, video consultation, chat mode, Whats App, SMS, and telemedicine via an approved EMR (Electronic Medical Record), where case records, images, investigations, tele-consultation recordings can be stored and retrieved.

Devices used for communication include Smartphones, desktop computer, laptop or tablet and patient monitoring devices located remotely that can be controlled with results obtained in real time.

\section{APPLICATIONS OF TELEMEDICINE IN HEALTH CARE}

Telemedicine can be applied in many ways in the field of health sector as follows: ${ }^{6}$

Tele-education: Distance learning with the help of telecommunication technologies, which are flexible and interactive.

Tele-health: Prevention, promotion and provision of health care facilities from a distance. It can be in the form of consultation or follow up.

Tele-home health: Telemedicine technology can be applied to provide home health care for elderly and homebound patients with chronic illness. It allows home healthcare professionals to monitor patients from a distance. A Computer Telephone Integrated (CTI) system can continuously monitor vitals of patients and transmit warning signals.

Disaster management: Telemedicine can play an important role to provide healthcare facilities to victims of disasters such as earthquake, tsunami, war, riots, etc. During disaster, most of the terrestrial communication links do not work properly or get damaged. A mobile and portable telemedicine system with satellite connectivity and customized telemedicine software is ideal for disaster relief in such circumstances. 


\section{ADVANTAGES OF TELEMEDICINE}

Advantages of telemedicine include the following: a) Telemedicine eliminates distance barrier and improves access to quality health services. b) Telemedicine provides easy, timely and equal accessibility to healthcare facilities to rural and remote areas, without decrease in quality of healthcare, thereby reducing inequity and barriers to access of health systems. ${ }^{7}$ c) Telemedicine facilitates patients and health care provider's access to specialists in health services. d) In emergency and critical care situations where moving a patient may not be feasible or undesirable, it is of immense help. e) It lessens the inconvenience and cost of patient transport and saves travel time and expenses for the patient and health care provider. f) Tele-home care might lead to quality home health care at reduced costs with greater patient satisfaction.

\section{TELEMEDICINE DURING COVID-19 PANDEMIC}

Despite its potential, telemedicine has not attained the 'boom' which it was meant to create and was not widely adopted until the recent outbreak of COVID 19 pandemic in 2020. The ongoing COVID-19 pandemic has caused a global crisis and several countries have taken containment measures such as lockdowns and curfews for several months to contain the spread of the virus. Health centres are overwhelmed with response efforts and health care institutions and health workers are redeployed to treat COVID-19 patients. This pandemic has forced healthcare institutions and government bodies to turn to alternative ways of providing healthcare while limiting exposure to the corona virus. The Covid-19 pandemic is causing several million people globally to try telemedicine for the first time. Government across all nations are investing in telemedicine to reduce patient load in emergency department during the COVID-19 pandemic. As the COVID-19 is causing havoc within the healthcare system, telemedicine is stepping up into the spotlight and helping healthcare providers to respond better to the needs of patients who need to touch base with their providers on the status of their health. Tele health is bridging the gap between people, physicians and health system and is emerging as an effective and sustainable solution for precaution, prevention and treatment of illnesses. Telemedicine is presenting itself as the ideal solution in this pandemic by limiting patient displacement to hospitals, allocating hospital capacity to important cases and curbing the disease spread to mass population and the medical staff on the frontlines. The American Academy of Family Physicians (AAFP) and the American Medical Association (AMA) have released telemedicine related guidelines. ${ }^{8} 9$ Medical council of India (MCI) has also announced guidelines to practice telemedicine by Registered Medical Practitioners. ${ }^{10}$ Both the Centre for Disease Control (CDC) and World Health Organization (WHO) are advocating telemedicine to monitor patients to reduce risk of spreading the virus by travelling to hospitals. ${ }^{11,12}$

\section{OBSTETRIC AND REPRODUCTIVE HEALTH CARE DURING THE COVID 19 PANDEMIC}

Globally, a large number of women are set out on a journey of parenthood amidst this COVID-19 pandemic. UNICEF has reported that an estimated 116 million babies will be born under the shadow of the COVID-19 pandemic. ${ }^{13}$ Access to appropriate healthcare is the key to better perinatal outcomes for the mother and baby. Pregnant women are traditionally advised to visit their health care providers multiple times throughout the pregnancy to receive expert care and to monitor the maternal and fetal wellbeing. However, due to the COVID-19 pandemic expecting mothers are currently unable to visit health centres due to the fear of getting infected with corona virus and missing out on emergency care due to the strained health services. There is a lot of fear and anxiety among pregnant women and there is a lot of uncertainty and unanswered queries regarding pregnancy and childbirth during this pandemic. In these circumstances, telemedicine can be a beneficial tool to both pregnant women and health care providers. This review outlines on how telemedicine can be effectively used in obstetric and reproductive health care during the COVID 19 pandemic.

\section{ROLE OF TELEMEDICINE IN PROVIDING OBSTETRIC HEALTH CARE SERVICES}

Telemedicine can facilitate a pregnant woman to have direct access to nursing support and health care providers, via online platforms or web-based apps. The web based apps may include information about specialists and health care providers, pregnancy education and appointment reminders. A study has reported that web based app use was associated with lower risk of delivering a low birth weight infant and a higher likelihood of completing antenatal care appointments. ${ }^{14}$ Thus using telemedicine services, patients may directly connect with doctors and nurses at times of need, while staying safe at home.

A broad range of pregnancy-related services can be offered via telemedicine. Consultation can be for addressing complaints for first time, regular antenatal checkup and postpartum follow up visits. Telemedicine can be used to provide antenatal care, home monitoring for conditions like diabetes and hypertension, consultation with specialists, mental healthcare services, postpartum care, lactation support and newborn care.

\section{Telemedicine for antenatal care}

Traditional antenatal care recommends that expecting mothers should have a number of in-person visits to the health care provider throughout pregnancy, demanding significant travel time and time off from their work or family. But only some antenatal visits truly require inperson care such as for getting ultrasound scans, lab investigations and vaccinations. Most of the antenatal visits are to provide patient education, answer patient 
queries and to monitor maternal vitals and fetal wellbeing. It has been reported that fewer antenatal visits are safe for low-risk pregnancies. ${ }^{15}$ Hence, health institutions and clinics can replace few of the antenatal inperson visits with telemedicine "virtual visits" via phone calls or videoconference. During tele- consultation, the health care provider should take a detailed obstetric history and should categorize the patient as low risk or high risk pregnancy based on history. Patients may be given clear instructions and supplies to monitor blood pressure, weight, fetal heart rate and fundal height at home or nearby clinic. The expecting mothers should be asked to transfer records of their body weight, blood pressure and daily fetal movement count recorded by self or in nearby health clinic. During teleconsultation, based on the period of gestation, women should be enquired regarding presence of any symptoms and signs related to abortion, eclampsia, gestational diabetes mellitus, antepartum hemorrhage and labor pains. Fundal height can be measured by caretaker of the patient after seeing demonstration videos. Health care provider can assess the severity of anemia by seeing for pallor of conjunctiva or tongue in video call and can prescribe hematinics. She can be motivated for successful delivery and future lactation. She can be educated about symptoms of onset of labor, danger signs of antenatal period and advised to contact immediately in case of emergency for early referral to tertiary care centre. These tele-consultation services allow patients to maintain continuity of care with their obstetricians, while staying at home during the COVD 19 pandemic.

High risk pregnancies with hypertension and diabetes can benefit from telemedicine through use of home monitoring devices for blood pressure and blood glucose. The values can be recorded and transmitted to their health care providers who can decide on whether in-person evaluation is indicated or not. Home monitoring of blood sugar in diabetic mothers may allow fewer visits to diabetes specialists, and improved health-related quality of life. ${ }^{16}$ It has been studied that telemedicine for blood sugar monitoring in women with gestational diabetes is as effective as the standard care, in achieving diabetic control during pregnancy with similar pregnancy outcomes. ${ }^{17,18}$ It has also been observed that home blood pressure monitoring in cases with hypertension in pregnancy was acceptable to most patients and could detect elevation in blood pressure. ${ }^{19,20}$ Home monitoring, in addition allows for active participation of patients in their self-care and promotes self-efficacy especially during hard times of COVID-19 pandemic.

With telemedicine services, patients and their health care providers can interact via videoconference or phone calls with maternal-fetal medicine (MFMs) specialists, genetic counselors, endocrinologists, nutritionist and cardiologists, without the need to travel to consult them in-person. Specialists can evaluate patients, review ultrasound imaging performed at a distance and recommend appropriate management plans. Telemedicine can thus increase the access to specialty care for patients who may otherwise forgo the specialist consult, in order to avoid travel fearing COVID 19 or due to lack of availability of specialists in their locality. Interaction with specialists encourages the health care providers to maintain quality care of pregnant women and facilitate their safe deliveries in nearby hospitals.

Pregnant women may require mental health services for mood disorders and substance abuse. Adequate medical and mental health support to antenatal women can be assured during this COVID-19 pandemic by providing access to mental health clinicians through online platforms. This increases the confidence of the expecting mothers on the medical system and strengthens the doctor- patient relationship.

\section{Telemedicine for postnatal care}

Postpartum care addresses the physical and emotional well-being of the patient after delivery, breastfeeding concerns, birth spacing and contraceptive needs and management of chronic diseases post-delivery. Traditionally, postpartum visits are scheduled at six weeks after delivery and ACOG now recommends post natal visit within three weeks of delivery. However, about $40 \%$ women do not attend any postpartum visit and statistics may worsen during the COVID-19 pandemic. Use of telemedicine in postpartum care could help address this issue and allows clients to interact with their health care providers and participate in virtual postpartum visits by phone or videoconference..$^{22,23}$

Patients can do home blood pressure and blood glucose monitoring during postpartum period and receive advise from their health care providers. Patient's queries regarding episiotomy or cesarean section wound care can be explained through videos, images or demonstration. Mothers can be advised regarding birth spacing, contraception and encouraged to opt for family planning methods. Mothers with breastfeeding difficulties can access online lactation consultants from their home. ${ }^{24}$

Mental tele-health services can be provided for postpartum depression and postpartum psychosis. $^{25}$ Systematic review on effectiveness of telemedicine to address maternal depression and postpartum mood disorders showed that cognitive behavioral therapy via telemedicine resulted in improvement of symptoms..$^{26,27}$

\section{Telemedicine for newborn care}

Wellness of newborn baby can be assessed by enquiring the mother or caretaker regarding adequate feeding, urine output, cry and activity of the baby. She can be educated about danger signs of baby and advised to contact immediately if needed for early referral of baby for direct care. It has been reported that parents viewed the telemedicine services as a lifeline, and saw it as a means of informing and guiding them, following early discharge 
from hospital after childbirth. ${ }^{23}$ Thus, telemedicine services has the potential for enhancing postnatal wellbeing of the mother and baby during the COVID-19 pandemic.

\section{TELEMEDICINE FOR REPRODUCTIVE HEALTH CARE SERVICES}

Telemedicine technologies may help address unmet reproductive health needs of women, particularly for rural areas and those with transportation barriers during COVID-19 pandemic. Telemedicine services could replace the need for in-person care in some reproductive health care services such as contraception, medication abortion, sexually transmitted infection (STI) care and sexual health. ${ }^{28}$ The modalities of delivery and levels of patient-provider interaction vary across these services. Services such as hormonal contraceptive prescriptions can be done via telemedicine. The patient shall provide a health history via a questionnaire or video consultation with a health care provider. The clinician may review the information remotely and determines eligibility for the required reproductive health care services. The patient may then receive the medications by pharmacy pick-up or mail. However for services including abortion care, lab STI testing, confirmatory testing and treatment of HIV, preventive services like pap smears and for pelvic examination, patients must still link to in-person care, thereby making telemedicine an adjunct to the existing health system in offering reproductive health services.

\section{BENEFITS OF TELEMEDICINE TO WOMEN SEEKING OBSTETRIC AND REPRODUCTIVE HEALTH CARE SERVICES DURING THE COVID- 19 PANDEMIC}

Benefits of telemedicine to women seeking obstetric and reproductive health care services during the COVID- 19 pandemic include the following: a) Patients can take less time off from work and family as they need not travel for routine in-person visits. b) Avoids sitting in the waiting room with potentially ill patients. c) Patients can get highquality health care while staying safe at home. d) Patients can access and send EMRs and messages to their doctors through secure systems. e) Increases patient engagement and continuity of care. f) Ensures supportive partnership between health care team and patients. g) Increases patient satisfaction.

\section{Limitations of telemedicine}

While telemedicine remains a promising tool to improve access to better obstetric, reproductive and newborn health during this COVID-19 pandemic, significant implementation challenges exist. Major barriers for telemedicine include limited affordability of low-income group to telecommunication tools, lack of knowledge about telecommunication and telemedicine, limited broad band internet accessibility in rural areas, questionable patient privacy, variable insurance coverage and high start-up investment for health care providers. These limitations can be overcome by combined multidisciplinary efforts of the government, telecommunication centers, health care providers, patients and the public. Government should take steps to regulate policies related to telemedicine and broaden its availability to underserved populations who could benefit from these technologies. Education of patients and public to improve their knowledge, attitude and practice of telemedicine service through mass media communication is absolutely necessary for effective telemedicine service.

\section{CONCLUSION}

Telemedicine can offer benefits over in-person care, including increased convenience, eliminating travel costs and allowing for more timely delivery of services. However, virtual visits can be challenging, especially for those with inadequate internet access and limited technology knowledge. Understanding the prevailing COVID-19 situation, patients and public can show their cooperation to Government by availing telemedicine services for their health needs while staying at home. This article underscores that telemedicine has a major role in providing effective healthcare not only during pandemic or disaster situation but also during routine practice of medicine.

Funding: No funding sources Conflict of interest: None declared Ethical approval: Not required

\section{REFERENCES}

1. Telemedicine-Opportunities and developments in member states. 2nd ed. Geneva, Switzerland: WHO press;2010.

2. History of Telemedicine-md Portal.md Portal. 2015. Available at: http://mdportal.com/education/historyof-telemedicine/. Accessed on 20 May 2020.

3. Marilyn JF. Telemedicine: a guide to assessing telecommunications in health care. Washington, D.C.: National Academy; 1996.

4. A Brief History of NASA's Contributions to Telemedicine. NASA. Available at: https://www.nasa.gov/content/a-brief-history-ofnasa-s-contributions-to-telemedicine/. Accessed on 20 May 2020.

5. Serper M. Current and future applications of telemedicine to optimize the delivery of care in chronic liver disease. Clin Gastroenterol Hepatol. 2018;16:157-61.

6. Mehta KG, Chavda P. Telemedicine: A boon and the promise to rural India. J Rev Prog. 2013;1:1-3.

7. Limor. Telemedicine Trends to Watch in 2018. Available at: Telemedicine.arizona.edu. Accessed on 20 May 2020.

8. Using telehealth to care for patients during the covid-19 pandemic. Available at: https:// 
www.aafp.org/ patient-care/emergency/2019. Accessed on 20 May 2020.

9. Robeznieks A. Key changes made to telehealth guidelines to boost COVID-19 care. Available at: https://www.ama-assn.org/delivering-care/publichealth/key-changes-made-telehealth-guidelinesboost-covid-19-care. Accessed on 18 May 2020.

10. Telemedicine practice guidelines enabling registered medical practitioners to provide healthcare using telemedicine. Board of Governors in supersession of the Medical Council of India. Appendix 5 of the Indian Medical Council Professional Conduct, Etiquette and Ethics Regulation, 2002. Accessed on 25 March 2020.

11. Preparing first responders, healthcare providers, and health systems CDC. Available at: https://www.cdc.gov/coronavirus/2019-ncov/cases. Accessed on 25 March 2020.

12. Home care for patients with suspected novel coronavirus (COVID-19) infection presenting with mild symptoms, and management of their contacts: interim guidance, 4 February 2020. Geneva: World Health Organization; 2020. Available at: https://apps.who.int/iris. Accessed on 25 March 2020.

13. Pregnant mothers and babies born during COVID19 pandemic threatened by strained health systems and disruptions in services UNICEF. Available at: https://www.unicef.org. Accessed on 7 May 2020.

14. Bush J, Barlow DE, Echols J, Wilkerson J, Bellevin K. Impact of a mobile health application on user engagement and pregnancy outcomes among wyoming medicaid members. Telemed Ehealth. 2017;23(11):891.

15. McDuffie RS, Beck A, Bischoff K, Cross J, Orleans M. Effect of frequency of prenatal care visits on perinatal outcome among low-risk women. A randomized controlled trial. JAMA. 1996.20;275(11):847-51.

16. Harisson TN. Sacks DA. Acceptability of virtual prenatal visit for women with getational diabetes mellitus. Women' Health Issues. 2017;9:351-5.

17. Dalfrà MG, Nicolucci A, Lapolla A. The effect of telemedicine on outcome and quality of life in pregnant women with diabetes. J Telemed Telecare. 2009; 15(5):238-42.

18. Ming WK, Mackillop LH, Farmer AJ, Loerup L, Bartlett K, Levy JC, et al. Telemedicine technologies for diabetes in pregnancy: a systematic review and meta-analysis. J Med Internet Res. 2016.9; 18(11):e290.
19. Heuvel JFM, Kariman SS, Solinge W, Franx A, Lely AT, Bekker MN. Feasibility study of a telemonitoring platform combining blood pressure and preeclampsia symptoms in pregnancy care. Eur J Obstet Gynecol Reprod Biol. 2019;240:226-s31.

20. Hoppe KK, Williams M, Thomas N, Zella JB, Drewry A, Kim K, et al. Telehealth with remote blood pressure monitoring for postpartum hypertension: a prospective single-cohort feasibility study. Pregnancy Hypertens. 2019;15:171-6.

21. Marnie J, Mooij MD, Des M. Nest: reimagining low-risk prenatal care. Mayo Clin Proceed. 2018;93(4):458-66.

22. Lavender T, Richens Y. Telephone support for women during pregnancy and the first six weeks postpartum. Available at: https:// doi.org/ 10.1002/ 14651858.CD009338.pub. Accessed on 18 May 2020.

23. Danbjørg RN, Wagner RN. Intervention among new parents followed up by an interview study exploring their experiences of telemedicine after early postnatal discharge. Midwifery. 2015;31(6):574-81.

24. Pines UL, Mehrotra A, Bogen DL. The emergence and promise of telelactation. Am J Obstet Gynecol. 2017;217(2):176-8.

25. Jean YK, Rockhill KM, Tong VT, Brian M, Sherry L. Trends in postpartum depressive symptoms 27 States, 2004, 2008, and 2012. Weekly. 2017;66(6):153-8.

26. Nair U, Armfield NR, Chatfield MD, Edirippulige $\mathrm{S}$. The effectiveness of telemedicine interventions to address maternal depression: a systematic review and meta-analysis. $\mathrm{J}$ Telemed Telecare. 2018;24(10):639-50.

27. Lee EW, Denison FC, Hor K, Reynolds RM. Webbased interventions for prevention and treatment of perinatal mood disorders: a systematic review. BMC Preg Childbirth. 2016;29:16-38.

28. Weigel G, Brittni F, Usha R. Telemedicine and Pregnancy Care. Women's Health Policy. Published: Feb 26, 2020. Available at: https:// www. kff.org/ womens-health-policy. Accessed on 25 March 2020.

Cite this article as: Sharmila V, Babu TA, Balakrishnan P. Optimizing telemedicine for providing obstetric and reproductive health care services during COVID-19 pandemic. Int J Community Med Public Health 2020;7:3278-83. 